\title{
Dynamic Process of Money Transfer Models
}

\author{
Yougui Wang* and Ning Ding \\ Department of Systems Science, School of Management, Beijing Normal \\ University, Beijing, 100875, People's Republic of China ygwang@bnu.edu.cn
}

\begin{abstract}
Summary. We have studied numerically the statistical mechanics of the dynamic phenomena, including money circulation and economic mobility, in some transfer models. The models on which our investigations were performed are the basic model proposed by A. Drăgulescu and V. Yakovenko [1], the model with uniform saving rate developed by A. Chakraborti and B.K. Chakrabarti [2], and its extended model with diverse saving rate [3]. The velocity of circulation is found to be inversely related with the average holding time of money. In order to check the nature of money transferring process in these models, we demonstrated the probability distributions of holding time. In the model with uniform saving rate, the distribution obeys exponential law, which indicates money transfer here is a kind of Poisson process. But when the saving rate is set diversely, the holding time distribution follows a power law. The velocity can also be deduced from a typical individual's optimal choice. In this way, an approach for building the micro-foundation of velocity is provided. In order to expose the dynamic mechanism behind the distribution in microscope, we examined the mobility by collecting the time series of agents' rank and measured it by employing an index raised by economists. In the model with uniform saving rate, the higher saving rate, the slower agents moves in the economy. Meanwhile, all of the agents have the same chance to be the rich. However, it is not the case in the model with diverse saving rate, where the assumed economy falls into stratification. The volatility distribution of the agents' ranks are also demonstrated to distinguish the differences among these models.
\end{abstract}

Key words: Transfer model, Dynamic Process, Money Circulation, Mobility

\section{Introduction}

Recently, wealth or income distribution has attracted much attention in the field of econophysics [4, 5, 6]. More than 100 years ago, Italian economist Pareto first found that the income distribution follows an universal power law [7]. However, the economy has undergone dramatic transitions in last century, some researchers had doubted about if the law still holds in the modern stage and turned to reexamine the income distribution and its shift by employing 
income tax data $[8,9,10,11,12]$. The empirical analysis showed that in many countries the income distribution typically presents with a power-law tail, and majority of the income distribution can be described by an exponential law. This universal shape of distribution and its shift trigger an increasing interests in exploring the mechanism behind them. To solve this problem, several multi-agent models have been developed by applying principles of statistical mechanics $[1,2,3,13,14,15]$. In these models, economic system is analogized to the ideal gas, where the agents can be regarded as particles, and money is just like energy. Therefore, the trading between agents can be viewed as collisions between particles in the gas. By using such analogy, the developed approach that applied to the ideal gas system now can be used to study this kind of economic system. Whatever the trading rule is set in these models, it is worthy noting that money is always transferred from one agent to another in the trading process. So this kind of models could be referred as money transfer models [16].

Leading the search into this issue was a paper by A. Drăgulescu and V. Yakovenko [1]. In their ideal-gas model, the economy is closed and the amount of money transferred in each round of trading is determined randomly. Their simulation analysis shows that the steady money distribution follows an exponential law. Several papers have extended the work by introducing different characteristics into the model and found that different trading rule may lead to different shapes of money distribution. A. Chakraborti and B.K. Chakrabarti examined the case where the agents do not take out all amount of money as they participate the exchange, but instead they save a part of their money [2]. This case is well grounded in reality, and the ratio they save is called saving rate hereafter. When the saving rate are the same for all agents, the money distribution obeys a Gamma law [17]. However, when the agents' saving rates are set randomly, the money distribution changes to a Power-law type [3]. A second extension looks at non-conservation. F. Slanina considered a case that the economy is not conserved but opened, and so he regarded it as inelastic granular gases [15]. Some further studies manage to seek for the exact mathematical solution by using a master equation [18, 19].

In fact, money transfer is a dynamic process. Besides the money distribution, it possess some other presentations. Thus, investigating the distribution only can not provide the whole picture of the relationship between the distribution and the trading rule. Some efforts have been put into the study on the dynamic mechanism behind the distribution, that opens more windows to observe how the economy works.

These works can be divided into two parts. One is about how the money moves in the assumed economy $[20,21,22]$. As we know, the money is not static even after the money distribution gets steady. They are always transferred among agents. Naturally, because of the randomness, whether in the simulations or in the reality, the time interval that money stays in one agent's pocket is a random variable which is named as holding time. The introduction 
of holding time opens a new path to understanding of the circulation velocity at micro level.

The other one is about how agents' positions shift in the economy [23]. Like the money, agents are not static in the transferring process. If the agents are sorted according to the amount of money they hold, it is found that the rank of any agent varies over time. This phenomenon is called mobility in economics. According to economists' argument, only analysis on the distribution is not sufficient especially when comparing the generating mechanism of income and the inequality $[24,25]$.

In addition, the study on the dynamic characters in the proposed models makes the evaluation criteria more complete. The aim of econophysicists to develop these models is to mimic the real economy by abstracting its essence. However, we cannot judge whether such abstraction is reasonable or not depending on the shape of distribution only. Thus, we must take the circulation and mobility into account when constructing a "good" multi-agent model.

In this paper, the dynamic processes of the transfer models are investigated by examining the holding time distribution and the degree of mobility. The models and simulations will be briefly presented in the next section. In the Sec. 3 and 4 , we will show the nature of circulation and mobility in these models respectively. Finally, we will give our conclusion in Sec. 5.

\section{Models and Simulations}

We start with the transfer model proposed by A. Drăgulescu and V. Yakovenko, in which the economic system is closed, put it in another way, the total amount of money $M$ and the number of economic agents $N$ are fixed. Each of agents has a certain amount of money initially. In each round of trading process, two agents $i, j$ are chosen to take part in the trade randomly. And it is also decided randomly which one is the payer or receiver. Suppose the amounts of money held by agent $i$ and $j$ are $m_{i}$ and $m_{j}$, the amount of money to be exchanged $\Delta m$ is decided by the following trading rule:

$$
\Delta m=\frac{1}{2} \varepsilon\left(m_{i}+m_{j}\right),
$$

where $\varepsilon$ is a random number from zero to unit. If the payer cannot afford the money to be exchanged, the trade will be cancelled. This model is very simple and extensible which is named as the basic model in this paper.

When A. Chakraborti and B.K. Chakrabarti intended to extend the basic model, they argued that the agents always keep some of money in hand as saving when trading. The ratio of saving to all of the money held is denoted by $s$ and called saving rate in this paper. For all the agents, the saving rates are set equally before the simulations. Like the trading pattern of the basic model, two agents $i, j$ are chosen out to participate the trade in each round. 
Suppose that at $t$-th round, agents $i$ and $j$ take part in trading, so at $t+1$-th round their money $m_{i}(t)$ and $m_{j}(t)$ change to

$$
m_{i}(t+1)=m_{i}(t)+\Delta m ; m_{j}(t+1)=m_{j}(t)-\Delta m,
$$

where

$$
\Delta m=(1-s)\left[(\varepsilon-1) m_{i}(t)+\varepsilon m_{j}(t)\right],
$$

and $\varepsilon$ is a random fraction. It can be seen that $\Delta m$ might be negative. That means agent $i$ is probably the payer of the trade. This model degenerates into the basic model if $s$ is set to be zero. In this model, all of agents are homogenous with the same saving rate. So we call it the model with uniform saving rate.

This model was further developed by B.K. Chakrabarti's research group by setting agents' saving rates randomly before the simulations and keeping them unchanged all through the simulations. Likewise, this is called the model with diverse saving rate. Correspondingly, the trading rule Equation (3) changes to

$$
\Delta m=\left(1-s_{i}\right)(\varepsilon-1) m_{i}(t)+\left(1-s_{j}\right) \varepsilon m_{j}(t),
$$

where $s_{i}, s_{j}$ are the saving rates of agent $i$ and $j$ respectively.

Our following investigations on the dynamic phenomena is based on these three models. The scale is the same for all the simulations: the number of agent $N$ is 1,000 and the total amount of money $M$ is 100,000 .

\section{Money Circulation}

As the medium of exchange, money is held and transferred by people. In the process of money transferring, if an agent receives money from others at one moment, he will hold it for a period, and eventually pays it to another agent. The time interval between the receipt of the money and its disbursement is named as holding time. We introduce the probability distribution function of holding time $P_{h}(\tau)$, which is defined such that the amount of money whose holding time lies between $\tau$ and $\tau+d \tau$ is equal to $M P_{h}(\tau) d \tau$. In the stationary state, the fraction of money $M P_{h}(\tau) d \tau$ participates in the exchange after a period of $\tau$. Then the average holding time can be expressed as

$$
\bar{\tau}=\int_{0}^{\infty} P_{h}(\tau) \tau d \tau .
$$

The velocity indicates the speed at which money circulates. Since money is always spent randomly in exchange, the transferring process can be deemed as a Poisson type, and the velocity of money can then be written as [20]

$$
V=\frac{1}{\bar{\tau}} .
$$


This is the statistical expression of the circulation velocity of money in terms of holding time.

Two caveats to this conclusion are in order. First, we need to observe the probability density function of holding time to check whether the transfer of money is a Poisson process. If the assumption is correct, the probability density function must take the following form

$$
P(\tau)=\lambda e^{-\lambda \tau},
$$

where $\lambda$ corresponds to the intensity of the Poisson process. We have carried out the measurement of holding time in our previous work [21]. In those simulations, the time interval between the moments when the money takes part in trade after $t_{0}$ for the first two times is recorded as holding time, supposing we start to record at round $t_{0}$. The data were collected after majority of money $(>99.9 \%)$ had been recorded and over 100 times with different random seeds.

The simulation results are shown in Fig.1. It can be seen the probability distributions of holding time decay exponentially in the model with uniform saving rate. This fact indicates that the process is a Poisson process. On the other case, when the saving rates are set diversely, the distribution changes to a power-law type.
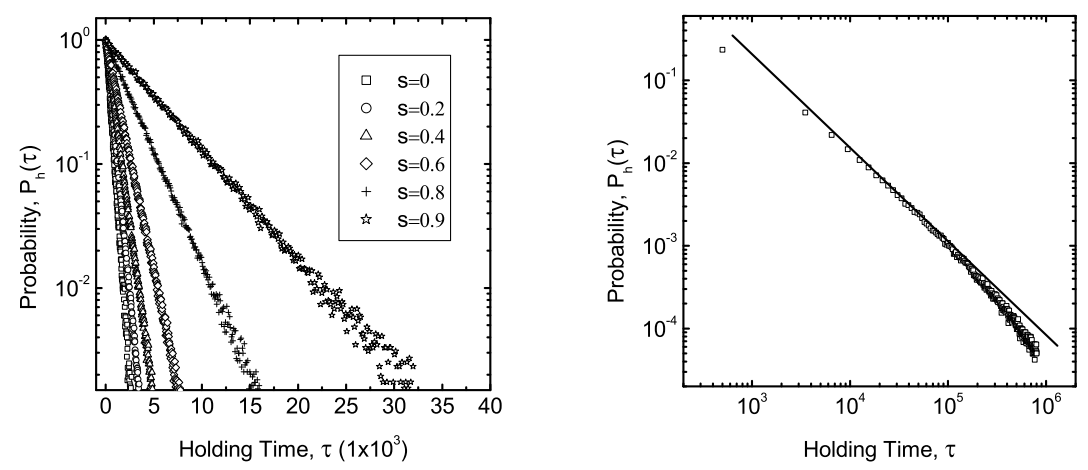

Fig. 1. The stationary distributions of holding time: (left panel) for the model with uniform saving rate in a semi-logarithmic scale, (right panel) for the model with diverse saving rate in a double-logarithmic scale, where the fitting exponent of the solid line is about -1.14 . Note that in the figure the probabilities have been scaled by the maximum probability respectively.

In the model with uniform saving rate, the monetary circulation velocity corresponds to the intensity of Poisson process, which is negatively related to the saving rate. Form Fig. 1 we can see that the lower the saving rate is, the 
steeper the distribution curve. This result is also plotted in Fig. 2, which tells us the relation between the velocity and the saving rate is not linear.

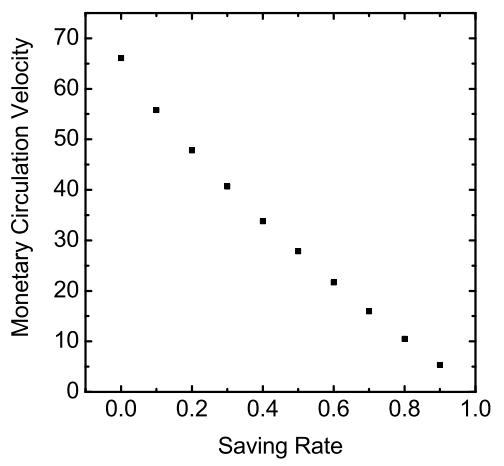

Fig. 2. The monetary circulation velocity versus the saving rate in the model with uniform saving rate.

Second, the relation between the velocity of money and the average holding time suggests that the velocity could be investigated by examining how economic agents make decisions on the holding time of money. There are many kinds of agents who may have different characters when they utilize money in an economic system, such as consumers, firms, and investors etc. We can choose one of them as a representative to examine how their spending decisions affect the velocity. The typical one is consumers whose behavior has always been depicted by the life-cycle model prevailed in economics. The model considers a representative individual who expects to live $T$ years more. His object is to maximize the lifetime utility

$$
U=\int_{0}^{T} u(C(t)) d t
$$

subject to the budget constraint condition

$$
\int_{0}^{T} C(t) d t \leq W_{0}+\int_{0}^{T} Y(t) d t
$$

where $u(\cdot)$ is an instantaneous concave utility function, and $C(t)$ is his consumption in time $t$. The individual has initial wealth of $W_{0}$ and expects to earn labor income $Y(t)$ in the working period of his or her life. The main conclusion deduced from this optimal problem is that the individual wants to smooth his consumption even though his income may fluctuate in his life time. From this conclusion, we can also calculate the average holding time of 
money based on the time path of income and consumption as the following form

$$
\bar{\tau}=\frac{\int_{0}^{T}[C(t)-Y(t)] t d t}{\int_{0}^{T} Y(t) d t} .
$$

With a few manipulations in a simple version of the life-cycle model [22], we get

$$
V=\frac{2}{T-T_{0}} .
$$

This result tells us that the velocity of money depends on the difference between the expected length of life $T$ and that of working periods $T_{0}$. It also implies that the velocity, as an aggregate variable, can be deduced from the individual's optimal choice. In this way, a solid micro foundation for velocity of money has been constructed.

\section{Economic Mobility}

It is the economists' consensus that static snapshots of income distribution alone is not sufficient for meaningful evaluation of wellbeing and the equality. This can be understood easily from a simple example. Suppose in an economy there are two individuals with money $\$ 1$ and $\$ 2$ initially. At the next moment, the amount of money held by them changes to $\$ 2$ and $\$ 1$. The distribution in this case is unchanged, but the ranks of both agents vary over time. Although the system seems unequal at either of the two moments in terms of the distribution, the fact is that the two individuals are quite equal combining these two moments. Besides, from this simple example, it can also been found that the structure of economy may vary heavily with an unchanged distribution. Thus the investigation on mobility is helpful not only to the measurement on equality but also to the exposure of the mechanism behind the distribution.

We investigated the mobility in the referred transfer models by placing emphasis on the "reranking" phenomenon. To show this kind of mobility, we sorted all of agents according to their money and recorded their ranks at the end of each round. All of data were collected after the money distributions get stationary and the sampling time interval was set to be 1000 rounds.

The time series of rank in these three models are shown in Fig.3. Then, we can compare the characters of rank fluctuation of these models. All of the agents in the basic model and the model with uniform saving rate can be the rich and be the poor. The rich have the probability to be poor and the poor also may be luck to get money to be the rich. The mobility in these two model are quite similar except the fluctuation frequency of the time series. The economy in the model with diverse saving rate is highly stratified (see Fig. 3c). The rich always keep their position, and the poor are doomed to be the poor. The agents in each level differ in their rank fluctuations. The higher the agent' rank, the smaller the variance of his rank. 

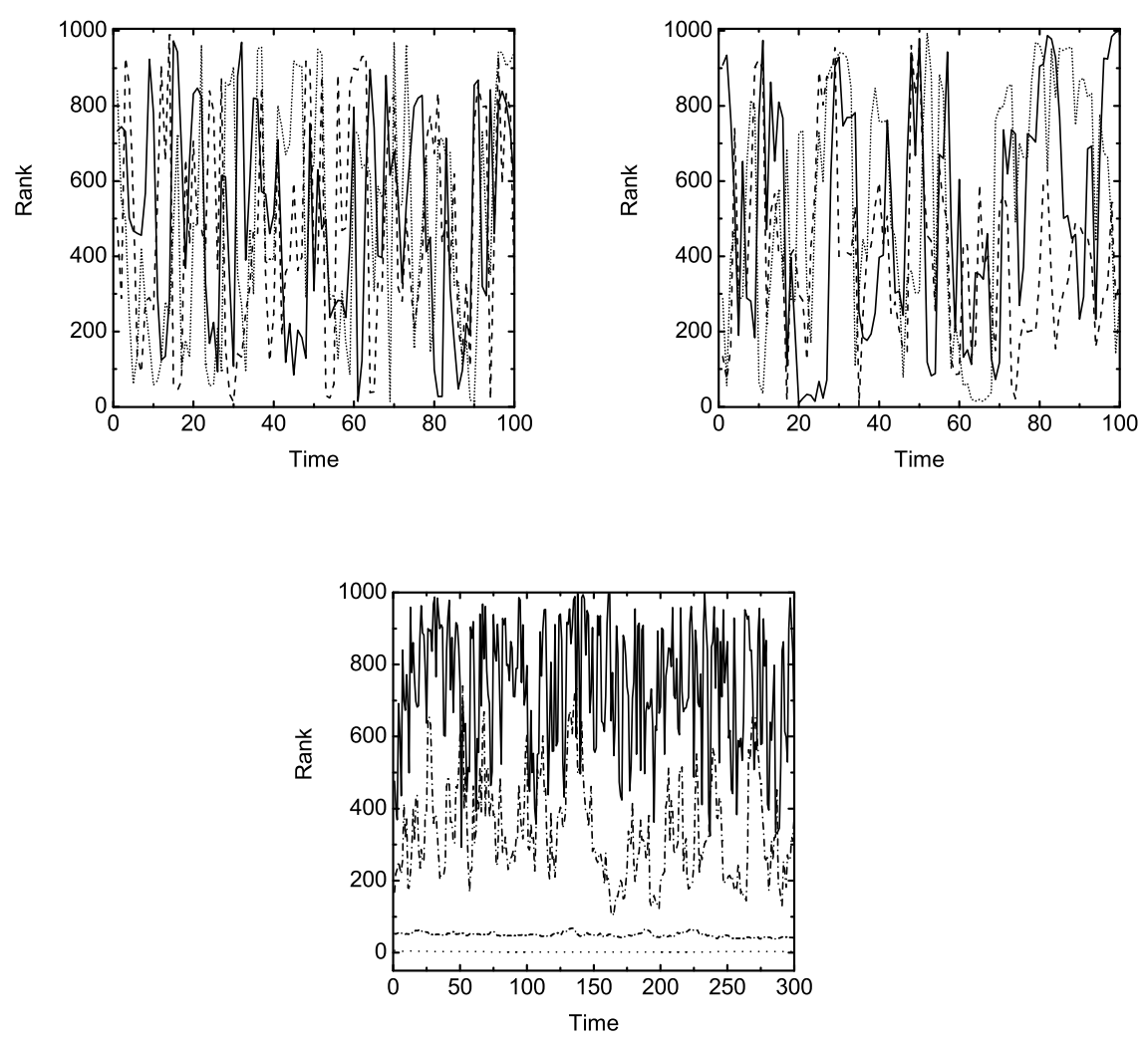

Fig. 3. The typical time series of rank (a) from basic model, (b) from the model with uniform saving rate $s=0.5$ and (c) from the model with diverse saving rate where the saving rates of these typical agents are $0.99853,0.9454,0.71548$ and 0.15798 (from bottom to top ) respectively.

Table 1. Comparison of the Three Transfer Models in Mobility

\begin{tabular}{c|c|c}
\hline & Mobility $l\left(t, t^{\prime}\right)$ & Stratification \\
\hline The Basic Model & 0.72342 & No \\
\hline The Model with Uniform Saving Rate & & No \\
$s=0.1$ & 0.70269 & \\
$s=0.3$ & 0.65165 & \\
$s=0.5$ & 0.58129 & \\
$s=0.7$ & 0.4773 & \\
$s=0.9$ & 0.30212 & \\
\hline The Model with Diverse Saving Rate & 0.19671 & Yes \\
\hline
\end{tabular}


To compare the mobilities quantitatively, we applied the measurement index raised by G. S. Fields et al [26]. The mobility between the two sample recorded in different moments is defined as

$$
l\left(t, t^{\prime}\right)=\frac{1}{N} \sum_{i=1}^{N}\left|\log \left(x_{i}(t)\right)-\log \left(x_{i}\left(t^{\prime}\right)\right)\right|,
$$

where, $x_{i}(t)$ and $x_{i}\left(t^{\prime}\right)$ are the rank of agent $i$ at $t$ and $t^{\prime}$ respectively. It is obvious that the bigger the value of $l$, the greater the degree of mobility. To eliminate the effect of the randomness, we recorded more than 9000 samples continuously and calculated the value of mobility $l$ between any two consecutive samples. The average value of $l \mathrm{~s}$ in these models are shown in Table 1. It can be found that the degree of mobility decreases as the saving rate
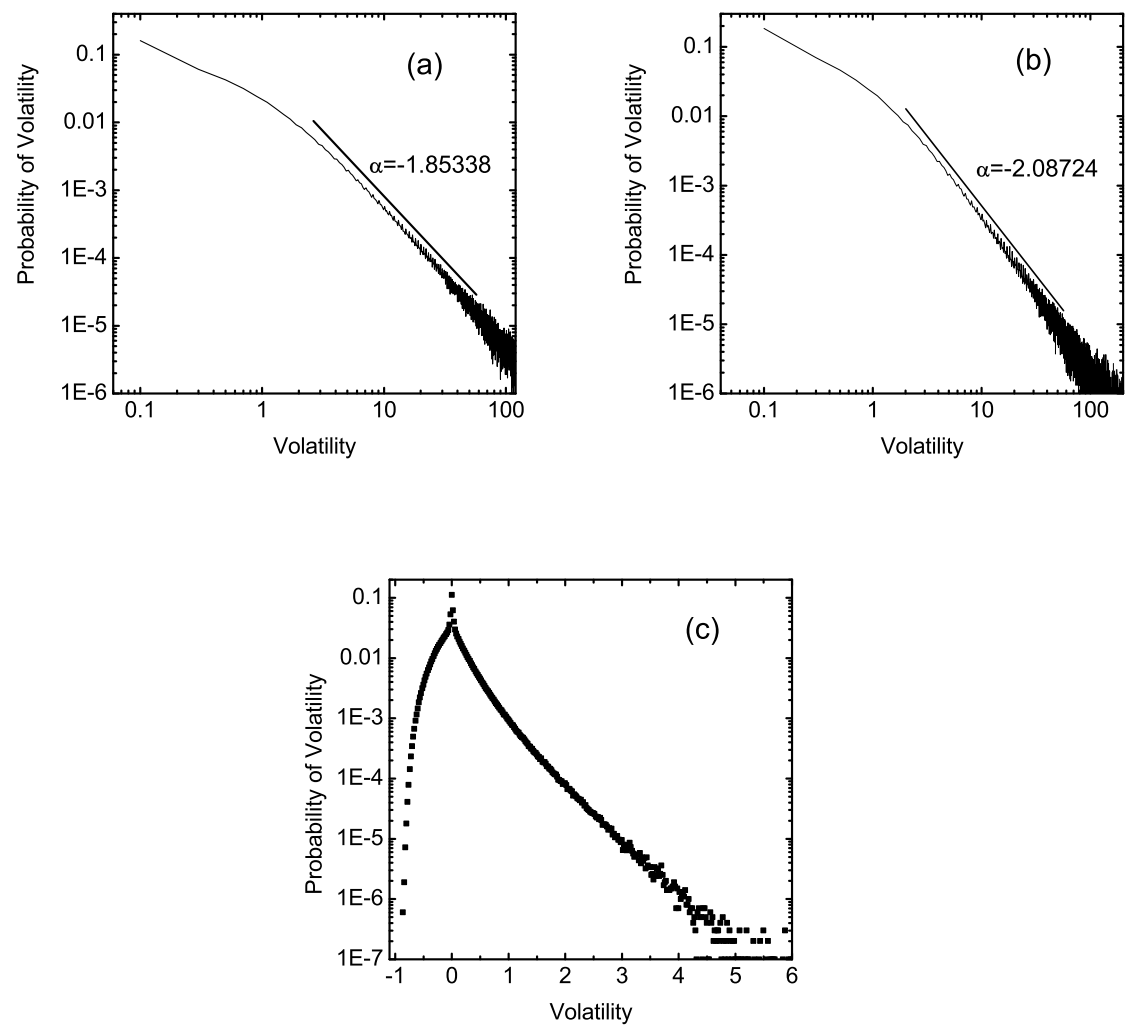

Fig. 4. The distribution of the volatility of agents' rank (a) for the basic model, (b) for the model with uniform saving rate $s=0.5$ and (c) for the model with diverse saving rate. 
increases in the model with uniform saving rate. The intuition for this result is straightforward. The larger the ratio agents put aside, the less money they take out to participate the trade. Then, the less money to lose or win. Thus, the higher saving rate, the less probability of change in rank or mobility. The very low degree of mobility in the model with diverse saving rate is due to its stratification.

To show more details of the mobility, we also obtain the distribution of the volatility $\left(\frac{x_{i}\left(t^{\prime}\right)-x_{i}(t)}{x_{i}(t)}\right)$ which is shown in Fig.4. It is noted that the distributions of the rank variety ratio are quite similar and follow power laws in the basic model and the model with uniform saving rate. The exponent of the power-law distribution is found to decrease as the saving rate increases. This phenomenon is consistent with the alter trend of the index because the higher the saving rate, the little money is exchanged and the smaller the volatility of rank. Consequently, when the saving rate increases, the right side of volatility distribution will shift to the vertical axis, leading to a more steeper tail. From Fig.4c, we can see that the volatility distribution in the model with diverse saving rate ends with an exponential tail as the times of simulations increase.

\section{Conclusion}

The dynamic phenomena of three transfer models, including money circulation and economic mobility, are presented in this paper. The holding time distributions in these models are demonstrated, and the relation between the velocity of money and holding time of money is expressed. Studies on this dynamic process lead us to a good understanding the nature of money circulation process and provide a new approach to the micro-foundation of the velocity. The "reranking" mobilities in these models are compared graphically and quantitatively. This observation provide more information about the dynamic mechanism behind the distribution. Such investigations suggest

that the characters of circulation and mobility should be considered when constructing a multi-agent model.

\section{References}

1. Drăgulescu A, Yakovenko VM (2000) Statistical mechanics of money. The European Physical Journal B 17:723-729

2. Chakraborti A, Chakrabarti BK (2000) Statistical mechanics of money: how saving propensity affects its distribution. The European Physical Journal B $17: 167-170$

3. Chatterjee A, Chakrabarti BK, Manna SS (2004) Pareto law in a kinetic model of market with random saving propensity. Physica A 335:155-163

4. Levy M, Solomon S (1996) Power laws are logarithmic Boltzmann laws. International Journal of Modern Physics C 7:595-601 
5. Bouchaud J-P, Mézard M (2000) Wealth condensation in a simple model of economy. Physica A 282:536-545

6. Scafetta N, Picozzi S, West BJ (2004) An out-of-equilibrium model of the distributions of wealth. Quantitative Finance 4:353-364

7. Pareto V (1897) Cours d'Economie Politique. Macmillan, Paris

8. Levy M, Solomon S (1997) New evidence for the power-law distribution of wealth. Physica A 242:90-94

9. Drăgulescu A, Yakovenko VM (2001) Evidence for the exponential distribution of income in the USA. The European Physical Journal B 20:585-589

10. Souma W (2001) Universal Structure of the personal income distribution. Fractals 9:463-470

11. Souma W, Fujiwara Y, Aoyamac H, Kaizoji T, Aoki M (2003) Growth and fluctuations of personal income. Physica A 321:598-604

12. Silva AC, Yakovenko VM (2005) Temporal evolution of the "thermal" and "superthermal" income classes in the USA during 1983-2001. Europhysics Letters 69:304-310

13. Ding N, Wang Y, Xu J, Xi N (2004) Power-law distributions in circulating money: effect of preferential behavior. International Journal of Modern Physics B 18:2725-2729

14. Hayes B (2002) Follow the money. American Scientist 90:400-405

15. Slanina F (2004) Inelastically scattering particles and wealth distribution in an open economy. Physical Review E 69:046102

16. Wang Y, Ding N, Xi N (2005) Prospects of money transfer models. In: Takayasu $\mathrm{H}$ (eds) Practical fruits of econophysics. Springer, Tokyo

17. Patriarca M, Chakraborti A, Kaski K (2004) Gibbs versus non-Gibbs distributions in money dynamics. Physica A 340:334-339

18. Patriarca M, Chakraborti A, Kaski K (2004) Statistical model with a standard Gamma distribution. Physical Review E 70:016104

19. Repetowicz P, Hutzler S, Richmond P (2004) Dynamics of Money and Income Distributions. arXiv: cond-mat/0407770

20. Wang Y, Ding N, Zhang L (2003) The circulation of money and holding time distribution. Physica A 324:665-677

21. Ding N, Xi N, Wang Y (2003) Effects of saving and spending patterns on holding time distribution. The European Physical Journal B 36:149-153

22. Wang Y, Qiu H (2005) The velocity of money in a life-cycle model. Physica A (in press)

23. Ding N, Xi N, Wang Y (2005) The economic mobility in money transfer models. Submitted to Physica A.

24. Kuznets SS (1966) Modern Economic Growth: Rate, Structure and Spread. Yale University, New Haven

25. Jarvis S, Jenkins SP (1998) How much income mobility is there in Britain? The Economic Journal 108:1-16

26. Fields GS, Ok E (1999) Measuring Movement of Incomes. Economica 66:455471 


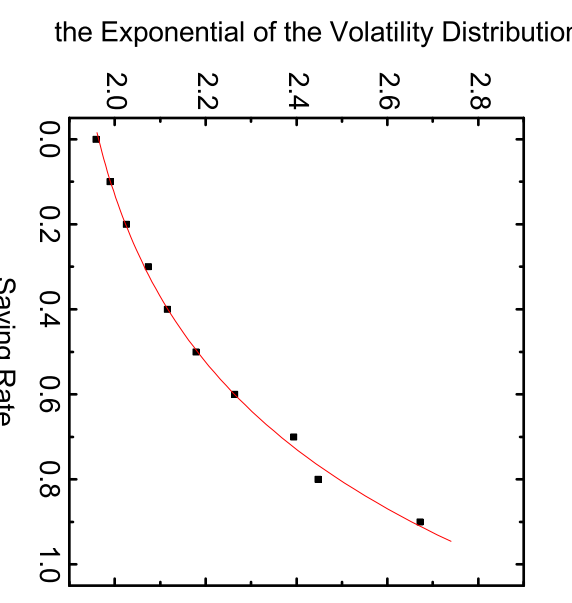

